International Journal of Bifurcation and Chaos, Vol. 11, No. 1 (2001) 215-224

(c) World Scientific Publishing Company

\title{
A SIMPLE APPROACH TO CALCULATION AND CONTROL OF UNSTABLE PERIODIC ORBITS IN CHAOTIC PIECEWISE-LINEAR SYSTEMS
}

\author{
TETSUSHI UETA \\ Department of Information Science, Tokushima University, \\ Tokushima, 770-8506 Japan \\ GUANRONG CHEN \\ University of Houston, Houston, TX 77204-4793, USA \\ TOHRU KAWABE \\ Institute of Information Sciences and Electronics, Tsukuba University, \\ Tsukuba, Ibaragi, 305-8573 Japan
}

Received October 1, 1999; Revised April 3, 2000

\begin{abstract}
This paper describes a simple method for calculating unstable periodic orbits (UPOs) and their control in piecewise-linear autonomous systems. The algorithm can be used to obtain any desired UPO embedded in a chaotic attractor, and the UPO can be stabilized by a simple state feedback control. A brief stability analysis of the controlled system is also given.
\end{abstract}

\section{Introduction}

It is well known that some simple electric circuits behave chaotically, such as the Chua's circuit [Madan, 1993] hysteresis circuits [Saito, 1990], the Colpitts oscillator [Kennedy, 1994] the buck converter [Yuan et al., 1998] and so on. In these systems, there exist some switching elements, so the overall system equation can be described by piecewise-linear functions.

For piecewise-linear systems since exact solutions are obtained as piecewise functions, many analytic issues such as the stability of attractors, bifurcation and existence of chaos can be discussed in a rigorous way. For example, Andronov studied the stability of the limit cycle observed from the piecewise-linear model of a vacuum-tube oscillator. This work is introduced in [Pontryagin, 1962], where the differential equation includes a step-wise (binary level) function which is approximated by some continuous saturation characteristics. Since this model is a second-order autonomous system, the stability of its limit cycle can be studied analytically. However, this particular piecewise-linear system is probably one of the very few examples whose dynamical properties can be obtained in an analytical way. The key point in this analysis is that the switching time, at which the system equation changes nonsmoothly, can be exactly evaluated. Unfortunately, calculating a periodic orbit and analyzing its stability are very difficult because the conditions on the switching times cannot be explicitly derived, especially for higher-order systems [Madan, 1993]. Therefore, one has to resort to appropriate numerical methods, particularly for higher-order systems, for this kind of computation and analysis. And yet, even if an efficient method is available for calculating periodic orbits, a large number of UPOs embedded within a chaos attractor are not able to calculate in general.

An attempt was made by Diakonos et al. [1998] to develop a systematic computational scheme for UPOs embedded within a chaotic attractor. Other efficient techniques to obtain UPOs are also 
available [Lathrop \& Kostelich, 1989; Ogorzalek, 1997]. Using a different approach, we present a simple method in this paper for the same purpose but only for piecewise-linear systems. This method utilizes the ergodic property of chaos and the characteristic of a wide basin of attraction of the Newton numerical method. More precisely, we first describe a simple numerical method for calculating periodic orbits (points) in piecewise-linear systems, then use Newton's method to provide numerical solutions for variational equations [Parker \& Chua, 1986, 1989]. This method requires no normal forms or approximations by continuous functions. To that end, we discuss an efficient algorithm for finding UPOs embedded within a specific chaotic attractor. This algorithm utilizes the ergodicity of chaos in addition to Newton's numerical method. Finally, we study a simple control technique that can stabilize these UPOs by using state feedback [Chen \& Dong, 1998, 1993]. Several examples of stabilizing UPOs are demonstrated, and a brief stability analysis is provided for completeness of the presentation.

\section{Calculating Periodic Orbits of a Piecewise-Linear System}

Consider a system of $m$ autonomous differential equations:

$$
\frac{d \mathbf{x}}{d t}=\mathbf{f}_{k}(\mathbf{x}) \quad k=0,1,2, \ldots, m-1,
$$

where $t \in \mathbf{R}$ is the time variable, $\mathbf{x} \in \mathbf{R}^{n}$ is the state vector. Assume that $\mathbf{f}_{k}$ is $C^{\infty}$ in all variables and parameters and that every equation in (1) has a unique solution for any finite initial value of $\mathbf{x}_{k}(0)$ such that

$\mathbf{x}_{k}(t)=\varphi_{k}\left(t, \mathbf{x}_{k}(0)\right), \mathbf{x}_{k} \equiv \mathbf{x}_{k}(0)=\varphi_{k}\left(0, \mathbf{x}_{k}(0)\right)$

Assume also that the function changes from $\mathbf{f}_{k}$ to $\mathbf{f}_{k+1}$ when a solution orbit $\boldsymbol{\varphi}_{k}$ starting from $\mathbf{x}_{k}(0)$ reaches a section $\Pi_{k+1}$ with $t=\tau_{k}$, where

$$
\Pi_{k+1}=\left\{\mathbf{x}_{k+1} \in \mathbf{R}^{n} \mid q_{k+1}\left(\mathbf{x}_{k+1}\right)=0\right\} .
$$

These sections are called break points. Suppose that a solution of (1) is written as follows:

$$
\begin{gathered}
\mathbf{x}_{k+1}(t)=\varphi_{k+1}\left(t, \mathbf{x}_{k+1}\right) \\
\mathbf{x}_{k+1}=\varphi_{k+1}\left(0, \mathbf{x}_{k+1}\right)=\varphi_{k}\left(\tau_{k}, \mathbf{x}_{k}\right) .
\end{gathered}
$$

Then we call this system a piecewise-smooth system [Bernardo \& Chen, 1999], especially if $\mathbf{f}_{k}$ are linear, we call the system a piecewise-linear system.

Clearly, a periodic orbit passing from $\Pi_{0}$ to $\Pi_{m-1}$ satisfies

$$
\mathbf{x}_{0}=\mathbf{x}_{m}=\varphi_{m-1}\left(\tau_{m-1}, \mathbf{x}_{m-1}\right) .
$$

Note that a solution of (1), given in the form of (4) and (5), is continuous but not differentiable on $\Pi_{k}$.

To investigate the characteristics of the system periodic solutions, we define the following local mappings:

$$
\begin{array}{rll}
T_{0}: & \Pi_{0} & \rightarrow \Pi_{1} \\
T_{1}: & \mathbf{x}_{0} & \mapsto \mathbf{x}_{1}=\varphi_{0}\left(\tau_{0}, \mathbf{x}_{0}\right) \\
& \rightarrow \Pi_{2} \\
\mathbf{x}_{1} & \mapsto \mathbf{x}_{2}=\varphi_{1}\left(\tau_{1}, \mathbf{x}_{1}\right) \\
& \cdots \\
T_{m-1}: & \Pi_{m-1} & \rightarrow \Pi_{0} \\
\mathbf{x}_{m-1} & \mapsto \mathbf{x}_{0}=\varphi_{m-1}\left(\tau_{m-1}, \mathbf{x}_{m-1}\right) .
\end{array}
$$

The Poincaré map is then defined as a differentiable map described by

$$
T=T_{0} \circ T_{1} \circ \cdots \circ T_{m-1} .
$$

Thus, the period $\tau$ of a limit cycle is given as

$$
\tau=\sum_{k=0}^{m-1} \tau_{k} .
$$

The derivative of the Poincaré map with respect to the initial state is calculated by a production of the Jacobian matrices obtained at each break point [Parker \& Chua, 1986]:

$$
\left.\frac{\partial T}{\partial \mathbf{x}_{0}}\right|_{t=\tau}=\left.\prod_{k=0}^{m-1} \frac{\partial T_{k}}{\partial \mathbf{x}_{k}}\right|_{t=\tau_{k}},
$$

where each Jacobian matrix can be written as

$$
\begin{aligned}
\left.\frac{\partial T_{k}}{\partial \mathbf{x}_{k}}\right|_{t=\tau_{k}} & =\frac{\partial \varphi_{k}}{\partial \mathbf{x}_{k}}+\frac{\partial \varphi_{k}}{\partial t} \frac{\partial \tau_{k}}{\partial \mathbf{x}_{k}} \\
& =\frac{\partial \varphi_{k}}{\partial \mathbf{x}_{k}}+\mathbf{f}_{k} \frac{\partial \tau_{k}}{\partial \mathbf{x}_{k}} .
\end{aligned}
$$

Since the function

$$
q_{k}\left(\mathbf{x}_{k}\right)=q_{k}\left(\boldsymbol{\varphi}_{k}\left(\tau_{k}, \mathbf{x}_{k}\right)\right)=0
$$


is differentiable, we have

$$
\frac{\partial q_{k}}{\partial \mathbf{x}_{k}}\left(\frac{\partial \varphi_{k}}{\partial \mathbf{x}_{k}}+\mathbf{f}_{k} \frac{\partial \tau_{k}}{\partial \mathbf{x}_{k}}\right)=\mathbf{0}
$$

where $q_{m}=q_{0}$. Thus, substituting (12) into (10) gives

$$
\frac{\partial T_{k}}{\partial \mathbf{x}_{k}}=\left[\mathbf{I}_{n}-\frac{1}{\frac{\partial q_{k}}{\partial \mathbf{x}} \cdot \mathbf{f}_{k}} \mathbf{f}_{k} \cdot \frac{\partial q_{k}}{\partial \mathbf{x}}\right] \frac{\partial \varphi_{k}}{\partial \mathbf{x}_{k}}
$$

where $\mathbf{I}_{n}$ is the $n \times n$ identity matrix, and $\partial \varphi_{k} / \partial \mathbf{x}_{k}$ is the fundamental matrix of the following variational equations:

$$
\begin{aligned}
& \frac{d}{d t}\left(\frac{\partial \varphi_{k}}{\partial \mathbf{x}_{k}}\right)=\frac{\partial \mathbf{f}_{k}}{\partial \mathbf{x}}\left(\frac{\partial \varphi_{k}}{\partial \mathbf{x}_{k}}\right) \\
& \left.\frac{\partial \varphi_{k}}{\partial \mathbf{x}_{k}}\right|_{t=0}=\mathbf{I}_{n}, \quad k=0,1,2, \ldots, m-1 .
\end{aligned}
$$

Note that this equation should be solved from $t=0$ to $t=\tau_{i}$, where $\tau_{i}$ is the time at which the orbit reaches the next section. Since the system is autonomous, we can reset $t$ to 0 at every section in the computing process. More precise mathematical treatments of these sections can be found in [Parker \& Chua, 1986].

Next, we define a local coordinate, $\mathbf{u} \in$ $\Sigma_{0} \subset \mathbf{R}^{n-1}$ corresponding to $\Pi_{0}$, by using a projection $p$ along with the associated embedding $\operatorname{map} p^{-1}$ :

$$
p^{-1}: \Sigma \rightarrow \Pi, \quad p: \Pi \rightarrow \Sigma .
$$

Accordingly, the Poincaré map in this local coordinate becomes

$$
\begin{aligned}
\mathcal{T}: \Sigma & \rightarrow \Sigma \\
\mathbf{u} & \mapsto p \circ T \circ p^{-1}(\mathbf{u}) .
\end{aligned}
$$

A fixed point of the Poincaré map is then obtained by solving the following equation:

$$
\mathcal{T}(\mathbf{u})-\mathbf{u}=\mathbf{0}
$$

Its Jacobian matrix, which is needed in Newton's method to be discussed below, is given by

$$
\begin{aligned}
D \mathcal{T}\left(\mathbf{u}_{k}\right)= & \frac{\partial p}{\partial \mathbf{x}} \frac{\partial T}{\partial \mathbf{x}_{0}} \frac{\partial p^{-1}}{\partial \mathbf{u}} \\
= & \prod_{k=0}^{m-1}\left(\frac{\partial p}{\partial \mathbf{x}} \frac{\partial T_{k}}{\partial \mathbf{x}_{k}} \frac{\partial p^{-1}}{\partial \mathbf{u}}\right) \\
= & \prod_{k=0}^{m-1}\left(\frac { \partial p } { \partial \mathbf { x } } \left[\mathbf{I}_{n}-\frac{1}{\frac{\partial q_{k}}{\partial \mathbf{x}} \cdot \mathbf{f}_{k}} \mathbf{f}_{k}\right.\right. \\
& \left.\left.\cdot \frac{\partial q_{k}}{\partial \mathbf{x}}\right] \frac{\partial \varphi_{k}}{\partial \mathbf{x}_{k}} \frac{\partial p^{-1}}{\partial \mathbf{u}}\right) .
\end{aligned}
$$

Finally, the fixed point is computed by using Newton's method with the initial condition $\mathbf{u}^{(0)}=$ $p\left(\mathbf{x}^{(0)}\right)$. The recurrence formula is written as follows:

$$
\left\{\begin{array}{l}
D \mathbf{F}\left(\mathbf{u}^{(i)}\right) \cdot \boldsymbol{\eta}^{(i)}=-\mathbf{F}\left(\mathbf{u}^{(i)}\right) \\
\mathbf{u}^{(i+1)}=\mathbf{u}^{(i)}+\boldsymbol{\eta}^{(i)}
\end{array} \quad i=01,2, \ldots\right.
$$

where

$$
\begin{gathered}
\mathbf{F}(\mathbf{u})=\mathcal{T}(\mathbf{u})-\mathbf{u}=\mathbf{0}, \\
D \mathbf{F}(\mathbf{u})=D \mathcal{T}(\mathbf{u})-\mathbf{I}_{n-1},
\end{gathered}
$$

and, as usual, $\boldsymbol{\eta}^{(i)}$ is solved at every iteration. When $\left\|\boldsymbol{\eta}^{(i)}\right\|_{\infty}<\epsilon$ for a predesired $\epsilon>0$, Newton's method is terminated.

The multipliers of the fixed point are calculated by solving the following characteristic equation:

$$
\left|\prod_{k=0}^{m-1} \frac{\partial \varphi_{k}}{\partial \mathbf{x}_{k}}\right|_{t=\tau_{k}}-\mu \mathbf{I}_{n} \mid=0
$$

The Runge-Kutta integration method is employed for solving the above variational equations as well as the original system (1) of differential equations. The initial step $\mathbf{u}^{(0)}$ from (1) is obtained by combining the Runge-Kutta scheme and the bisection method on the corresponding local section (break point) [Parker \& Chua, 1986].

We should emphasize that taking local coordinate $\mathbf{u}$ is mandatory. If we take $\mathbf{F}(\mathbf{x})=T(\mathbf{x})-\mathbf{x}=$ 0 instead of Eq. (22), the variable $\mathbf{x}$ should lie on the section by the condition of the Poincare section $q_{0}\left(\mathbf{x}_{0}\right)=0$. This means $\mathbf{x}$ is not independent, 
and this fact affects the convergence of the Newton method.

\section{Computing UPOs Embedded in a Chaotic Attractor}

If one has a good approximation of the periodic point on the local section, the recurrence formula Eq. (21) will converge and the accurate location of the periodic point is identified. It is well known in numerical simulations on chaotic systems, however, one can only visualize stable solutions such as stable periodic orbits, while unstable orbits such as saddle points are difficult to display or exactly calculated. Thus, to compute an UPO using the above algorithm, we have to resort to utilize the information about the unstable orbits of the system. The main problem is how to provide the first-guess for the recurrence formula. We suggest to utilize the ergodicity of the chaotic dynamics, which are generally disregarded in numerical computation. In so doing, we gain quite a lot of first-guess information for using the recurrence formula.

The algorithm is summarized as follows:

Step 1. Choose the parameter in which the system behaves chaotically, and set an appropriate initial condition. Select a number $m$ defined in Eq. (5), where $m$ indicates how many times the orbit hits different points on the sections.

Step 2. Calculate the orbit by solving Eq. (1) with the Runge-Kutta scheme. The Poincaré mapping point should be calculated by using the bisection method, and using the map $n$ times.

Step 3. Iterate the recurrence formula Eq. (21) (then Eqs. (1) and (14) are solved simultaneously). If the formula converges, print the periodic point $\mathbf{u}_{0}$; if the formula is not convergent within a few iterations, stop the iteration of the formula and, regardless of success or failure of the formula, go to Step 2.

The calculated points must be unstable and the whole family of UPOs may be produced by the Runge-Kutta method started from these points.

Compared to the method of Diakonos et al. [1998], our method is simpler and convenient to use, which utilizes the ergodicity of chaos, i.e. it is possible for its orbit to visit the neighborhood of every UPO. Note that the main advantage is that the sta- bility issue of the orbit is by-passed, which is usually quite troublesome. Moreover, Newton's method has quadratic convergence, so that we can expect a wide basin of attraction for the computation in general.

\section{Computing UPOs in Chua's Circuit}

\subsection{Problem description and calculation of UPOs}

Here, we show an example of obtaining an UPO embedded within the chaotic attractor of Chua's circuit.

The system is described by the following differential equations:

$$
\begin{aligned}
& \dot{x}^{1}=\alpha\left(-x^{1}+x^{2}-H\left(x^{1}\right)\right):=f^{0} \\
& \dot{x}^{2}=x^{1}-x^{2}+x^{3}:=f^{1} \\
& \dot{x}^{3}=-\beta x^{2}:=f^{2},
\end{aligned}
$$

where

$$
H(x)= \begin{cases}a x+a-b, & x \leq-1 \\ b x, & |x|<1 \\ a x-a+b, & x \geq 1\end{cases}
$$

By using the expression Eq. (1), each $\mathbf{f}_{k}$ can be described as follows:

$$
\begin{aligned}
& \mathbf{f}_{0}=\left\{\begin{array}{l}
-\alpha(a+1) x^{1}+\alpha x^{2}+\alpha(b-a)=f_{0}^{0} \\
x^{1}-x^{2}+x^{3}=f_{0}^{1} \\
-\beta x^{2}=f_{0}^{2},
\end{array}\right. \\
& \mathbf{f}_{1}=\left\{\begin{array}{l}
-\alpha(b+1) x^{1}+\alpha x^{2}=f_{1}^{0} \\
x^{1}-x^{2}+x^{3}=f_{1}^{1} \\
-\beta x^{2}=f_{1}^{2},
\end{array}\right. \\
& \mathbf{f}_{2}=\left\{\begin{array}{l}
-\alpha(a+1) x^{1}+\alpha x^{2}+\alpha(a-b)=f_{2}^{0} \\
x^{1}-x^{2}+x^{3}=f_{2}^{1} \\
-\beta x^{2}=f_{2}^{2} .
\end{array}\right.
\end{aligned}
$$

In view of the characteristics of the nonlinear term $H$, it is clear that we must place the local sections at $x^{1}= \pm 1$. Thus, the state space is segmented into three half-regions in $\mathbf{R}^{3}$.

$\Gamma_{1}\left\{\mathbf{x} \in \mathbf{R}^{3} \mid x^{1}<-1\right\}, \quad \Gamma_{2}\left\{\mathbf{x} \in \mathbf{R}^{3} \mid-1 \leq x^{1} \leq 1\right\}$

and

$$
\Gamma_{3}\left\{\mathbf{x} \in \mathbf{R}^{3} \mid x^{1}>1\right\} .
$$

In the following, for simplicity we discuss the case where the intended UPO passes only $\Gamma_{1}$ and $\Gamma_{2}$. 
Naturally, we can define a local section by the scalar function

$$
q(\mathbf{x})=q_{0}=q_{1}=x+1=0
$$

and provide $\Pi_{0}$ at which the periodic flow from $\Gamma_{1}$ reaches $\Gamma_{2}$. Therefore, the associated projection and the embedding are:

$$
\begin{aligned}
p: \mathbf{R}^{3} & \rightarrow \mathbf{R}^{2} \\
\left(x^{1}, x^{2}, x^{3}\right) & \mapsto\left(u^{1}, u^{2}\right)=\left(x^{2}, x^{3}\right), \\
p^{-1}: \mathbf{R}^{2} & \rightarrow \mathbf{R}^{3} \\
\left(u^{1}, u^{2}\right) & \mapsto\left(x^{1}, x^{2}, x^{3}\right)=\left(-1, u^{1}, u^{2}\right),
\end{aligned}
$$

where we always let $x^{1}=-1$. The Jacobians for these maps are

$$
\frac{\partial p}{\partial \mathbf{x}_{k}}=\left(\begin{array}{ccc}
0 & 1 & 0 \\
0 & 0 & 1
\end{array}\right) \quad \text { and } \quad \frac{\partial p^{-1}}{\partial \mathbf{u}_{k}}=\left(\begin{array}{ll}
0 & 0 \\
1 & 0 \\
0 & 1
\end{array}\right) .
$$

Similarly,

$$
\frac{\partial q}{\partial \mathbf{x}_{k}}=\left(\begin{array}{lll}
1 & 0 & 0
\end{array}\right)
$$

Thus, we have

$$
\frac{d q}{d \mathbf{x}_{k}} \cdot \mathbf{f}_{k}=f_{k}^{0}
$$

and

$$
\mathbf{f}_{k} \cdot \frac{d q}{d \mathbf{x}_{k}}=\left(\begin{array}{ccc}
f_{k}^{0} & 0 & 0 \\
f_{k}^{1} & 0 & 0 \\
f_{k}^{2} & 0 & 0
\end{array}\right)
$$

Consequently,

$$
I_{3}-\frac{1}{\frac{d q}{d \mathbf{x}_{k}} \cdot \mathbf{f}_{k}} \cdot \mathbf{f}_{k} \cdot \frac{d q}{d \mathbf{x}_{k}}=\left(\begin{array}{ccc}
0 & 0 & 0 \\
-f_{k}^{1} / f_{k}^{0} & 1 & 0 \\
-f_{k}^{2} / f_{k}^{0} & 0 & 1
\end{array}\right)
$$

The remaining calculation of (20) is the variations $\partial \varphi_{k} / \partial \mathbf{x}_{k}$. They are computed from (14) by using the Runge-Kutta method. Its Jacobian matrix of $\mathbf{f}_{k}$ is given by

$$
\begin{aligned}
& \frac{\partial \mathbf{f}_{0}}{\partial \mathbf{x}_{0}}=\left(\begin{array}{ccc}
-\alpha(a+1) & \alpha & 0 \\
1 & -1 & 1 \\
0 & -\beta & 0
\end{array}\right), \\
& \frac{\partial \mathbf{f}_{1}}{\partial \mathbf{x}_{1}}=\left(\begin{array}{ccc}
-\alpha(b+1) & \alpha & 0 \\
1 & -1 & 1 \\
0 & -\beta & 0
\end{array}\right) .
\end{aligned}
$$

We fix the parameters as: $\alpha=9.0, \beta=100 / 7$ $(\approx 14.28571429), a=-5 / 7(\approx-0.71428571), b=$ $-8 / 7(\approx-1.14285714)$. There is a double scroll attractor using this set of parameter values, as shown in Fig. 1.

Applying the Newton's method formulated in Eq. (21), a pair of UPOs that cross the sections twice $(m=2)$ are calculated, as shown in Figs. 2(a) and 2(b). These orbits are originally stable at $\alpha<$ 8.2 , and are calculated by the continuation method [Parker \& Chua, 1989]. We can also calculate them by using the method discussed in Sec. 3 with $m=2$ and $\epsilon=10^{-12}$ specified therein. The location of the fixed point is also shown in Table 1 . The eigenvalues of this fixed point are: $\left(\mu_{1}, \mu_{2}, \mu_{3}\right)=$ $(0.999998,-3.219297,-0.004119)$, and the period is $\tau=2.425509$.

Due to the symmetry of the system, these two attractors are symmetric with respect to the origin so that if one of them is obtained, the other can be obtained by simply inverting the sign of each coordinate. Nevertheless, the algorithm can detect both of them individually.

Figures 2(c) and 2(d) show the orbits with $m=4$ and Figs. 2(e)-2(h) show the orbits with $m=8$, which clearly wanders between two areas. Figures 2(i) $-2(\mathrm{n})$ show the orbits with $m=8$, which travel through every $\Gamma_{1}-\Gamma_{3}$ area. Moreover, Figs. 2(o) $-2(\mathrm{w})$ show the orbits with $m=18$, and Fig. 2(x) shows the orbit with $m=20$.

In the numerical simulation, these orbits with the initial condition shown in Table 1 can be seen as a stable orbit for a while but eventually it collapses and is absorbed by the chaotic attractor.

The accuracy of the multipliers are sensitive to the step size $h$ of the Runge-Kutta method. This is

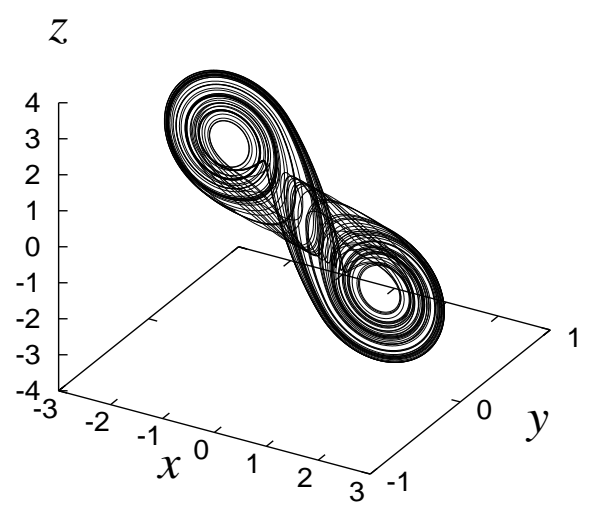

Fig. 1. A double scroll attractor. $\alpha=9, \beta=100 / 7$, $a=-5 / 7, b=-8 / 7$. 
Table 1. A list of unstable periodic points.

\begin{tabular}{lcl}
\hline No. & $m$ & \multicolumn{1}{c}{ Location } \\
\hline (a) & 2 & $(1.0,0.249220818156,-0.382555213770)$ \\
(b) & 2 & $(-1.0,0.340911528961,1.413657311515)$ \\
(c) & 4 & $(-1.0,0.254159440024,1.063931210592)$ \\
(d) & 4 & $(1.0,0.055077194872,-0.138652507702)$ \\
(e) & 8 & $(-1.0,0.359790197110,1.487480328560)$ \\
(f) & 8 & $(1.0,-0.359790196654,-1.487480327354)$ \\
(g) & 8 & $(-1.0,0.374633124347,1.539840997444)$ \\
(h) & 8 & $(1.0,-0.374633124347,-1.539840997444)$ \\
(i) & 8 & $(1.0,0.093582377853,-0.107383838370)$ \\
(j) & 8 & $(1.0,0.127104744082,-0.448588074837)$ \\
(k) & 8 & $(-1.0,0.271242138920,1.131274519567)$ \\
(l) & 8 & $(-1.0,0.259036328538,1.084397263536)$ \\
(m) & 8 & $(-1.0,0.389678369333,1.602575686019)$ \\
(n) & 8 & $(-1.0,0.389847777644,1.601231613985)$ \\
(o) & 18 & $(-1.0,0.388208969456,1.594470694615)$ \\
(p) & 18 & $(1.0,-0.383448856631,-1.575204563904)$ \\
(q) & 18 & $(1.0,-0.240180118818,-1.008823320661)$ \\
(r) & 18 & $(1.0,-0.278983608935,-1.161793509221)$ \\
(s) & 18 & $(1.0,-0.378707856965,-1.560866384193)$ \\
(t) & 18 & $(1.0,-0.375425039983,-1.543004675104)$ \\
(u) & 18 & $(-1.0,0.257982140023,1.079000894966)$ \\
(v) & 18 & $(-1.0,0.389787554925,1.602978857083)$ \\
(w) & 18 & $(1.0,-0.364528334894,-1.505968657391)$ \\
(x) & 20 & $(1.0,-0.229638853919,-0.967268270405)$ \\
\hline & & \\
\hline
\end{tabular}

perhaps a significant feature of the piecewise-linear systems. In the case of a pure nonlinear system, very accurate values of multipliers can be obtained using $h=0.01$. If a more accurate solution is desired, a small value of $h$ should be used. However, there is a lower limit for $h$ in the Runge-Kutta method. The choice of $h$ is not comparatively affected by the convergence rate of Newton's method. If more accurate data or the verification of the existence for UPOs are needed, the interval in Newton's method is good to use [Galias, 1998].

\subsection{Control of UPOs}

Chen and Dong [1993] proposed a method for stabilizing UPOs by a unified canonical feedback. Bernardo and Chen [1999] also investigated applications of such feedback control technique for piecewise-smooth systems. In this approach, the controlled system is written as

$$
\begin{aligned}
\frac{d \mathbf{x}}{d t} & =\mathbf{f}_{k}(\mathbf{x})+\mathbf{K}(\hat{\mathbf{x}}-\mathbf{x}) \\
k & =0,1,2, \ldots, m-1,
\end{aligned}
$$

where $\hat{\mathbf{x}}$ is the target UPO. By using this feedback, one can stabilize any UPO calculated by the previously described algorithm. If one provides a signal generator which can store the data of several UPOs, the controlled system can stabilize a specified UPO from the chaotic region. Although some detailed stability analyses have already been given in [Chen \& Dong, 1993; Bernardo \& Chen, 1999], we show another analysis in this section. The stability of the feedback is confirmed only by the characteristic equations, a Lyapunov function or relevant criterion are not necessary. Note that the Poincaré mapping must be required to calculate UPOs, however, it is not needed in the control process.

Now we discuss a simple sufficient condition for this control strategy. For convenience, we consider a UPO crossing two half-regions:

$$
\begin{aligned}
\dot{\mathbf{x}}(t)= & \mathbf{A}_{0} \mathbf{x}(t)+\mathbf{P} \quad \text { if } \quad \mathbf{x} \in \Gamma_{1}, \\
& \Gamma_{1}:\left\{\mathbf{x} \in \mathbf{R}^{3} \mid x^{1}<-1\right\} \\
\dot{\mathbf{x}}(t)= & \mathbf{A}_{1} \mathbf{x}(t) \quad \text { if } \quad \mathbf{x} \in \Gamma_{2}, \\
& \Gamma_{2}:\left\{\mathbf{x} \in \mathbf{R}^{3} \mid-1<x^{1}<1\right\},
\end{aligned}
$$

where, $\mathbf{x}=\left(x^{1}, x^{2}, x^{3}\right)^{\top}$ and

$$
\begin{aligned}
\mathbf{A}_{0}= & \left(\begin{array}{ccc}
-\alpha(a+1) & \alpha & 0 \\
1 & -1 & 1 \\
0 & -\beta & 0
\end{array}\right) \\
\mathbf{A}_{1}= & \left(\begin{array}{ccc}
-\alpha(b+1) & \alpha & 0 \\
1 & -1 & 1 \\
0 & -\beta & 0
\end{array}\right), \\
\mathbf{P} & =\left(\begin{array}{c}
\alpha(b-a) \\
0 \\
0
\end{array}\right)
\end{aligned}
$$

where $\alpha, \beta, a, b$ are constants. Both $\mathbf{A}_{0}$ and $\mathbf{A}_{1}$ have a pair of stable complex eigenvalues and a real but unstable eigenvalue. Also, $\mathbf{x}_{0}(t)$ and $\mathbf{x}_{1}(t)$ are solutions of Eq. (39) satisfying

$$
\begin{aligned}
& \mathbf{x}_{0}(t)=\varphi_{0}\left(t, \mathbf{x}_{0}(0)\right), \\
& \mathbf{x}_{1}(0)=\mathbf{x}_{0}\left(\tau_{0}\right)=\varphi_{0}\left(\tau_{0}, \mathbf{x}_{0}(0)\right) . \\
& \mathbf{x}_{1}(t)=\varphi_{1}\left(t, \mathbf{x}_{1}(0)\right), \\
& \mathbf{x}_{0}(0)=\mathbf{x}_{1}\left(\tau_{1}\right)=\varphi_{1}\left(\tau_{1}, \mathbf{x}_{1}(0)\right) .
\end{aligned}
$$




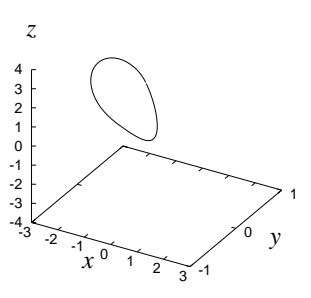

(a)

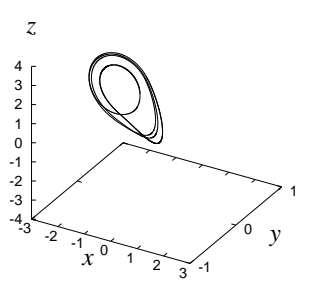

(e)

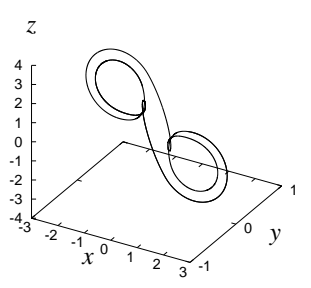

(i)

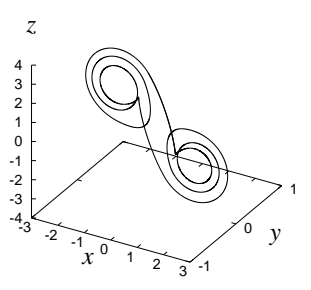

$(\mathrm{m})$

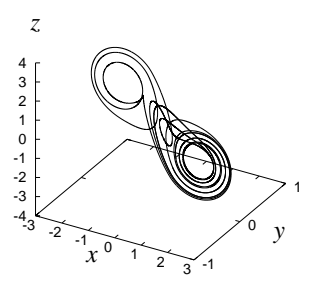

(q)

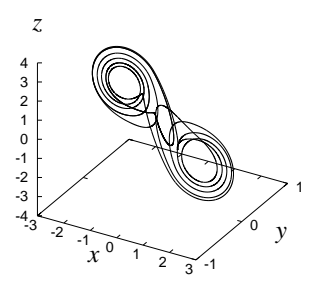

(u)

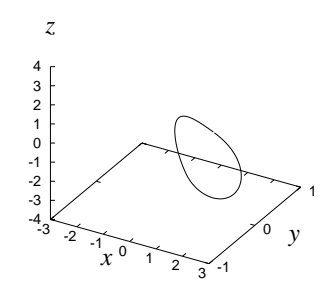

(b)

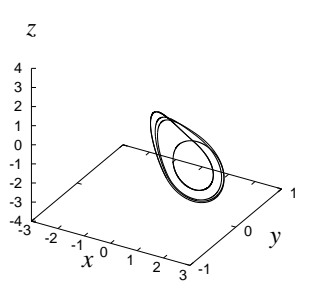

(f)

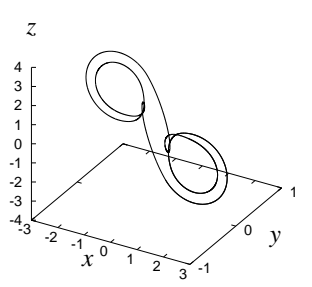

(j)

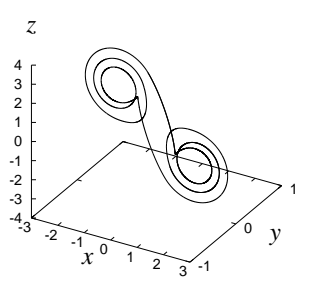

(n)

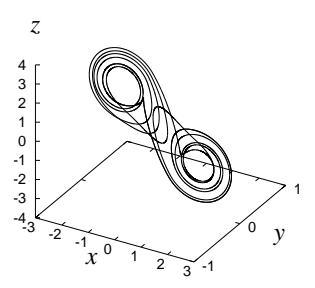

(r)

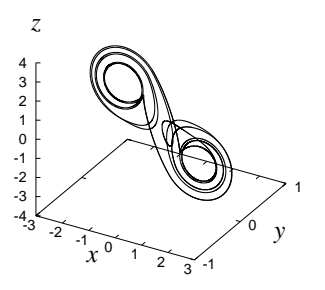

(v)

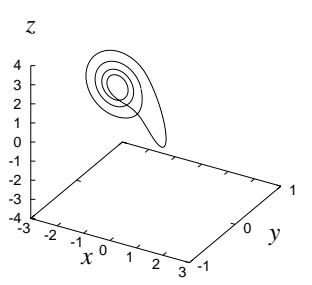

(c)

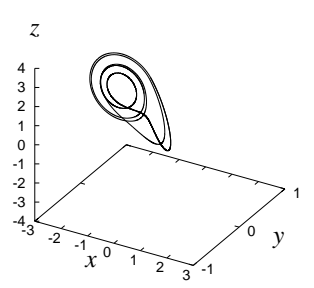

(g)

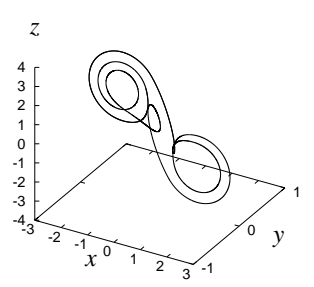

(k)

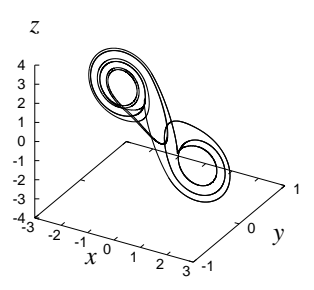

(o)

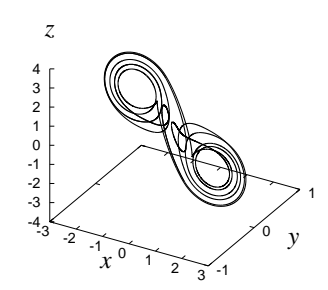

(s)

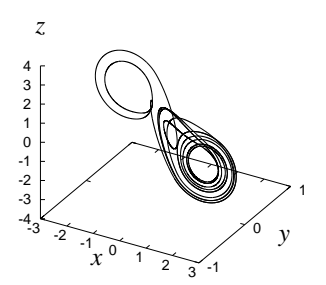

(w)

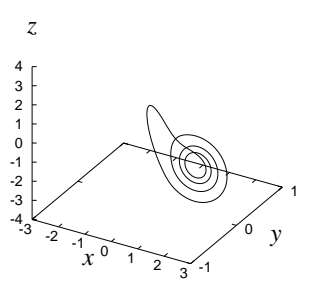

(d)

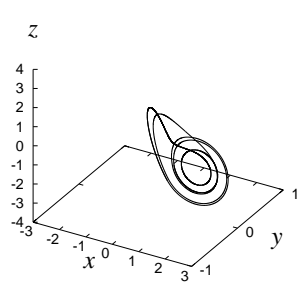

(h)

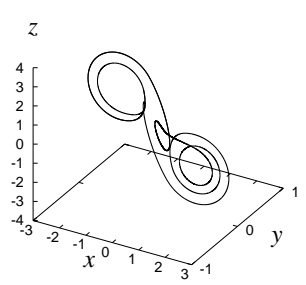

(1)

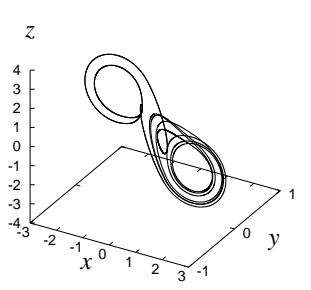

(p)

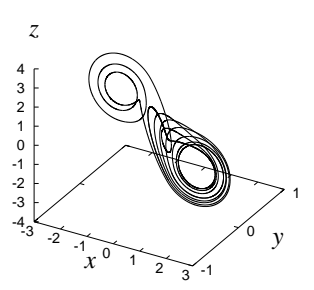

(t)

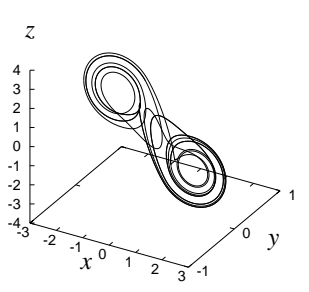

(x)

Fig. 2. Unstable periodic orbits in the double scroll attractor. $\alpha=9, \beta=100 / 7, a=-5 / 7, b=-8 / 7$. 
Each of $\varphi_{0}(t)$ and $\varphi_{1}(t)$ forms a periodic orbit. We rewrite these piecewise orbits as $\hat{\mathbf{x}}_{0}(t)$ and $\hat{\mathbf{x}}_{1}(t)$.

Now we assume that $\hat{\mathbf{x}}_{0}(t)$ and $\hat{\mathbf{x}}_{1}(t)$ form an unstable periodic solution under appropriate parameter values. We try to stabilize this orbit by using the following state feedback:

$$
\begin{array}{ll}
\dot{\mathbf{x}}(t)=\mathbf{A}_{0} \mathbf{x}(t)+\mathbf{P}+\mathbf{K}\left(\hat{\mathbf{x}}_{0}(t)-\mathbf{x}(t)\right) & \text { if } x^{1}<-1, \hat{x}^{1}<-1 \\
\dot{\mathbf{x}}(t)=\mathbf{A}_{1} \mathbf{x}(t)+\mathbf{K}\left(\hat{\mathbf{x}}_{0}(t)-\mathbf{x}(t)\right) & \text { if } x^{1}>-1, \hat{x}^{1}<-1 \\
\dot{\mathbf{x}}(t)=\mathbf{A}_{1} \mathbf{x}(t)+\mathbf{K}\left(\hat{\mathbf{x}}_{1}(t)-\mathbf{x}(t)\right) & \text { if } x^{1}>-1, \hat{x}^{1}>-1 \\
\dot{\mathbf{x}}(t)=\mathbf{A}_{0} \mathbf{x}(t)+\mathbf{P}+\mathbf{K}\left(\hat{\mathbf{x}}_{1}(t)-\mathbf{x}(t)\right) & \text { if } x^{1}<-1, \hat{x}^{1}>-1,
\end{array}
$$

where we use

$$
\mathbf{K}=\operatorname{diag}\left\{K_{11}, K_{22}, K_{33}\right\} .
$$

Firstly we consider the first and the third cases of Eqs. (45):

$$
\begin{aligned}
& \dot{\mathbf{x}}_{0}(t)=\mathbf{A}_{0} \mathbf{x}_{0}(t)+\mathbf{P}+\mathbf{K}\left(\hat{\mathbf{x}}_{0}(t)-\mathbf{x}_{0}(t)\right), \\
& \dot{\mathbf{x}}_{1}(t)=\mathbf{A}_{1} \mathbf{x}_{1}(t) \mathbf{K}\left(\hat{\mathbf{x}}_{1}(t)-\mathbf{x}_{1}(t)\right)
\end{aligned}
$$

where $\hat{\mathbf{x}}_{0}$ and $\hat{\mathbf{x}}_{1}$ satisfy Eqs. (39) within each $\Gamma_{i}$, $i=1,2$; therefore,

$$
\begin{aligned}
\dot{\hat{\mathbf{x}}}_{0}(t) & =\mathbf{A}_{0} \hat{\mathbf{x}}_{0}(t)+\mathbf{P} \\
\dot{\hat{\mathbf{x}}}_{1}(t) & =\mathbf{A}_{1} \hat{\mathbf{x}}_{1}(t)
\end{aligned}
$$

Since the system is piecewise linear, the variation around the periodic solution at any instant $t$ is written as

$$
\begin{aligned}
& \mathbf{x}_{0}(t)=\hat{\mathbf{x}}_{0}(t)+\xi(t), \\
& \mathbf{x}_{1}(t)=\hat{\mathbf{x}}_{1}(t)+\zeta(t) .
\end{aligned}
$$

Substituting these equation into Eqs. (47) and then removing the relationship Eqs. (48) from the result, we have

$$
\begin{aligned}
& \dot{\xi}(t)=\left(\mathbf{A}_{0}-\mathbf{K}\right) \xi(t), \\
& \dot{\zeta}(t)=\left(\mathbf{A}_{1}-\mathbf{K}\right) \zeta(t) .
\end{aligned}
$$

Suppose, in a simple design, that $K_{11}=K_{22}=$ $K_{33}=K$. Then the characteristic equations for Eqs. (50) become

$$
\begin{aligned}
& \operatorname{det}\left[\mathbf{A}_{0}-(\mu+K) I\right]=0, \\
& \operatorname{det}\left[\mathbf{A}_{1}-(\mu+K) I\right]=0 .
\end{aligned}
$$

If we take a sufficiently large positive value for $K$, then all eigenvalues of the matrices $\mathbf{A}_{0}-\mathbf{K}$ and $\mathbf{A}_{1}-\mathbf{K}$ become negative real parts since all coefficients of the characteristic equations can be regulated by $K$.

Similarly, we have the following equations from the second and the fourth cases of Eqs. (45):

$$
\begin{aligned}
& \dot{\xi}(t)=\left(\mathbf{A}_{0}-\mathbf{K}\right) \xi(t)+\mathbf{K}\left(\hat{\mathbf{x}}_{1}(t)-\hat{\mathbf{x}}_{0}(t)\right) \\
& \dot{\zeta}(t)=\left(\mathbf{A}_{1}-\mathbf{K}\right) \zeta(t)+\mathbf{K}\left(\hat{\mathbf{x}}_{0}(t)-\hat{\mathbf{x}}_{1}(t)\right) .
\end{aligned}
$$

The remainders $\hat{\mathbf{x}}_{1}(t)-\hat{\mathbf{x}}_{0}(t)$ and $\hat{\mathbf{x}}_{0}(t)-\hat{\mathbf{x}}_{1}(t)$ are bounded for all $t$, because $\hat{\mathbf{x}}_{0}(t)$ and $\hat{\mathbf{x}}_{1}(t)$ are part of a periodic solution. Here, solutions of the variational equations (52) are bounded. Thus the control goal is achieved by choosing a sufficiently large value for $K$. For the cases of other UPOs passing through every half-region with appropriate order, the stability analysis can be considered in the same way.

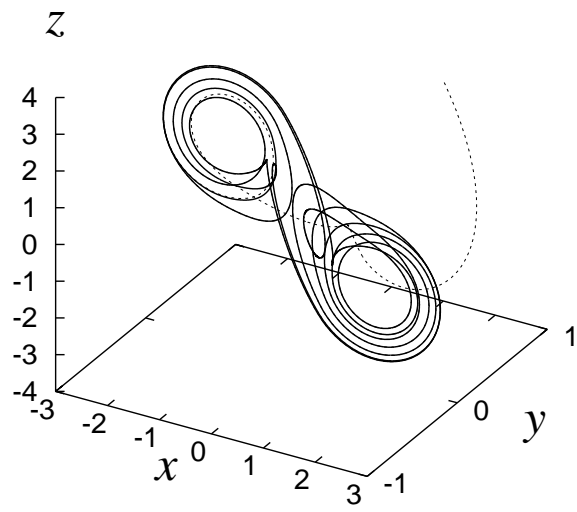

(a)

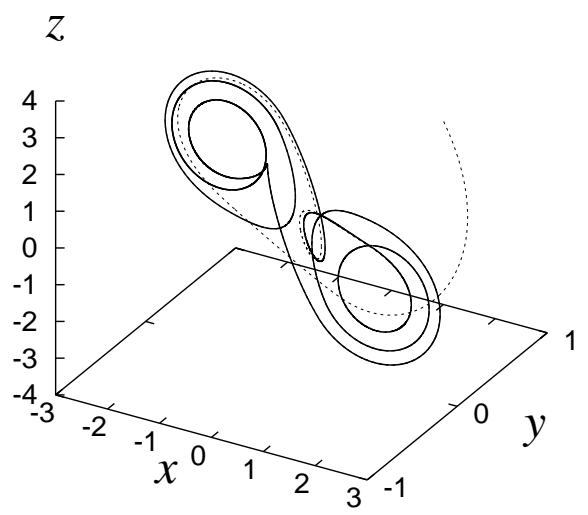

(b)

Fig. 3. Simulation results of the controlled Chua's circuit. (a) $m=10$, the initial point is $(1,1,1)^{\top}$, (b) $m=20$, the initial point is $(1,1,2)^{\top}$. The dashed and solid lines show the transient trajectory and the stabilized UPO, respectively. 


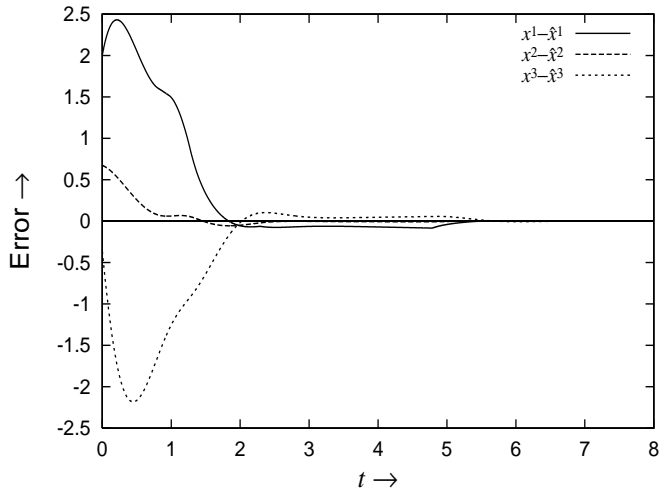

(a)

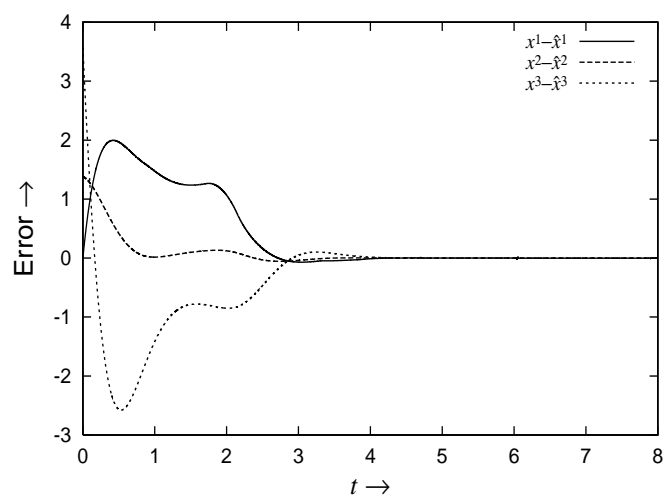

(b)

Fig. 4. Time response of errors according to Figs. 3(a) and $3(\mathrm{~b})$.

As illustrated examples of the control strategy described by Eq. (38), we show a couple of simulations for UPOs calculated at Sec. 2. Figures 3(a) and 3(b) show simulation results of chaos stabilization for UPOs with $m=10$ and 20, respectively. We chose a gain matrix as $\mathbf{K}=\operatorname{diag}\{2,2,2\}$. The initial point is $(1,1,1)^{\top}$ and $(1,1,2)^{\top}$, respectively. The reference UPO signals were sampled 30,000 points per cycle.

$\mathrm{Xu}$ and Bishop proposed a self-locating control of UPOs [Xu \& Bishop, 1996]. It is a stabilizing method based on the OGY method with Newton's algorithm. In contrast to this, our method provides automatic detection of UPOs by utilizing ergodicity of chaos and Newton's algorithm. Any parameter value is not be perturbed.

\section{Conclusions}

We have proposed a simple yet efficient algorithm for calculating periodic orbits of piecewise-linear systems, particularly the unstable periodic orbits embedded within a chaotic attractor. As an application, we have also discussed the stabilization of a calculated UPO from a chaotic region by designing a state feedback controller, along with some brief stability analysis on the controlled system.

\section{Acknowledgments}

The authors would like to thank Dr. Inaba of Utsunomiya University, Dr. Komuro of Teikyo University of Science and Technology, Dr. Nakajima of Kinki University, and Prof. Kawakami and Dr. Kousaka of Tokushima University for their insightful suggestions and fruitful discussions.

\section{References}

Bernardo, M. \& Chen, G. [1999] "Controlling bifurcations in nonsmooth dynamical systems," in Controlling Chaos and Bifurcations in Engineering Systems, ed. Chen, G. (CRC press), pp. 391-415.

Chen, G. \& Dong, X. [1993] "Controlling Chua's circuit," J. Circuits Syst. Comput. 3(1), 139-149.

Chen, G. \& Dong, X. [1998] From Chaos to Order: Methodologies, Perspectives and Applications (World Scientific, Singapore).

Diakonos, F. K., Schmelcher, P. \& Biham, O. [1998] "Systematic computation of the least unstable periodic orbits in chaotic attractors," Phys. Rev. Lett. 81(20), 4349-4352.

Duchesne, L. [1993] "Using characteristic multiplier loci to predict bifurcation phenomena and chaos - a tutorial," IEEE Trans. Circuits Syst. CAS-I-40(10), 683-688.

Galias, Z. [1998] "Investigations of periodic orbits in electronic circuits with interval Newton method," Proc. 1998 IEEE Int. Symp. Circuits Syst. III, pp. 370-373.

Kennedy, M. P. [1994] "Chaos in the Colpitts oscillator," IEEE Trans. Circuits Syst. CAS-41(11), 771-774.

Lathrop, D. P. \& Kostelich, E. J. [1989] "Characterization of an experimental strange attractor by periodic orbits," Phys. Rev. A40(7), 4028-4031.

Madan, R. N. [1993] Chua's Circuit: A Paradigm for Choas (World Scientific, Singapore).

Ogorzalek, M. J. [1997] Chaos and Complexity in Nonlinear Electronic Circuits (World Scientific, Singapore).

Parker, T. S. \& Chua, L. O. [1986] "Efficient solution of the variational equation for piecewise-linear differential equations," J. Circuit Th. Appl. 14 305-314.

Parker, T. S. \& Chua, L. O. [1989] Practical Numerical Algorithms for Chaotic Systems (Springer-Verlag, NY).

Pontryagin, L. S. [1962] Ordinary Differential Equations (Addison-Wesley, NY). 
Saito, T. [1990] "An approach toward higher dimensional hysteretic chaos generators," IEEE Trans. CAS37(3), 399-409.

Xu, D. \& Bishop, S. R. [1996] "Self-locating control of chaotic systems using Newton algorithm," Phys. Lett. A210, 273-278.
Yuan, G., Banerjee, S., Otto, E. \& Yorke, J. A. [1998] "Border-collision bifurcations in the buck converter," IEEE Trans. Circuits Syst. CAS-I-45(7), 707-716. 